\title{
AURAL: Robots, Evolution and Algorithmic Composition
}

\author{
Artemis Moroni ${ }^{1}$, Jônatas Manzolli ${ }^{2}$, Mariana Shellard ${ }^{2}$ \\ ${ }^{1}$ CTI Renato Archer, Robotics and Computer Vision Division, Rod. D. Pedro I, km 143,6, \\ 13069-901, Campinas, SP, Brazil \\ artemis.moroniecti.gov.br \\ ${ }^{2}$ Unicamp, Interdisciplinary Nucleus of Sound Studies, Rua da Reitoria, 165, \\ Cidade Universitária Zeferino Vaz, 13083-872, Campinas, SP, Brazil \\ jonatas@nics. unicamp.br, marianashellard@gmail.com
}

\section{Extended Abstract}

Focusing on the interactiviness that a robotic interface establishes between the virtual and the real world, some sensory systems and mobile robotic platforms were developed for the AURAL project, a robotic evolutionary environment for sound production $[1,2]$.

In the AURAL environment, the sonification is generated by an evolutionary mapping of the robot trajectories into sound events. One issue addressed is the structure/novelty tradeoff [3]. By applying these ideas to algorithmic composition systems means that more knowledge and structure allows the creation of new pieces that are more tightly matched to the desired musical genre. However, the flipside of more structure is less new material. The highly constrained output will be less likely to stray beyond a genre's limitations or it may be surprising. Thus, the highly structured composition system will be less general, able to reach less 'music space' with its output.

In the AURAL, this tradeoff is treated by creating an interplay between sound, real-world artifacts, user and behavioral information. Through the interaction among the evolutionary sound process, the artificial vision system and the mobile robots [4]. The sound interface has a Graphic Area, the heart of the system, wherein the user may draw curves to be sent as trajectories to the robots. This area is associated with a conceptual sound space with two axis, the "red" one, or melodic, and the "blue" one, or rhythmic. The paths travelled by the robots in the arena are observed by the artificial vision system and sent, as sequences of points, to the sonification module. The red curves, sent as trajectories to the robots and the blue curves associated with the paths travelled guide the evolutionary sound process across different regions in the sound space.

AURAL was presented in an art gallery where the visitors could appreciate the sound output and the interaction among the robots, as a kind of choreography. The visitors drew curves in the graphic area, which were transmitted as trajectories to a master robot, the Nomad. While the robots (until 4) moved in the arena, virtually traveling along the conceptual sound space, people changed the orchestra, rhythm and 
pitch controls, investigating the sound possibilities. Both a process of man-machine interaction and parallel exploration occurred.

On the last day of the exhibition, a dancer, three musicians and the AURAL system itself, with four robots, performed an interactive concert called Robotic Variations. The performance of the robots was accompanied by the musicians, who knew the type of music that would be generated, but they had to be able to adapt the performance. At the same time, the dancer, tracked by her red hat, was interacting with the robots, all interfering in the music that was being generated.

One can see as an interesting aspect of the AURAL environment the possibility of different setups to explore distinct levels of interaction among humans and machines. AURAL supplies a platform for robotic experimentation and artistic creation exploring human and machine interaction and bringing about results that could not be obtained otherwise.

\section{Acknowledgements}

We thank the students Thiago Spina, Eddy Nakamura, Felipe Augusto and Helen Fornazier, who worked with Pioneer, Nomad, Roomba and iCreate robots. We also thank the students Lucas Soares, Igor Dias, Igor Martins, Eduardo Camargo and Flavio Kodama who worked in the development of OmniEye and JaVOX. We thank the musicians Cesar Traldi, Chiquinho Costa, Adriano Monteiro and the dancer Tatiana Benone, who played in the performance at Unicamp/IA Art Gallery. We especially thank Adriana Giarola Kayama for her commitment to the narration of this video. We thank the Scientific Initiation Program of the National Research Council (PIBIC/CNPq), the Center for Technology Information Renato Archer and the Interdisciplinary Nucleus for Sound Studies of the State University of Campinas (NICS/UNICAMP) for making this research possible. This research work is part of the AURAL project, supported by the Foundation for the Research in São Paulo State (FAPESP) process 05/56186-9. Manzolli is supported by the Brazilian Agency CNPq. This work is licensed under the Creative Commons [CC-BY].

\section{References}

1. Moroni, A., Manzolli, J., Von Zuben, F. J., Gudwin, R.: VoxPopuli: An Interactive Evolutionary System for Algorithmic Music Composition. Leonardo Music Journal 10, 49-542 (2000)

2. Moroni, A., Manzolli, J., Von Zuben, F. J., Gudwin, R.: Evolutionary Computation for Music Evolution. In: Bentley, P., Corne, D. (eds.) Creative Evolutionary Systems, pp. 205-221, Morgan Kaufmann, San Francisco (2002)

3. Todd, P. M., Werner, G. M.: Frankensteinian Methods for Evolutionary Music Composition. Musical Networks: Parallel Distributed Perception and Performance, Cambridge: The MIT Press, 313-340 (1999)

4. Moroni, A., Manzolli, J.: From Evolutionary Composition to Robotic Sonification. In: Di Chio, C. et al. (eds.) EvoApplications 2010. LNCS 6025, pp. 400-410, Springer, Heidelberg (2010) 